\title{
Eine Metasynthese zu Lehrwerkanalysen von regionalen DaF- Lehrwerken *
}

\author{
Adalet Uyan (iD), Ankara \\ https://dx.doi.org/10.37583/diyalog.845595
}

\begin{abstract}
Deutsch)
Das Ziel dieser Studie ist es, das präsente Wissen zu Lehrwerkanalysen von regionalen DaF-Lehrwerken in einer Metasynthese systematisch zusammenzufassen und überblicklich als Ganzes darzustellen. Das Forschungsinteresse dieser Arbeit wurde durch die Fragen geleitet, welche gemeinsamen Themen- und Forschungsbereiche sich binnen den Primärstudien aufweisen lassen und welche Aspekte in Analysen von regionalen DaF-Lehrwerken häufig/ weniger häufig thematisiert werden. Aus den Ergebnissen der Synthese lässt sich ableiten, dass die in den Primärstudien analysierten regionalen DaF-Lehrwerke am häufigsten auf den Aspekt Landeskunde \& interkulturelles Lernen untersucht werden. Analysen zur Wortschatzvermittlung, zu Selbstorganisationsformen und Vermittlungskonzepten sind in den eingeschlossenen Studien nur gering vertreten.
\end{abstract}

Schlüsselwörter: regionale DaF-Lehrwerke, Lehrwerkanalyse, Metasynthese, Lehrbuchanalyse, Deutschlehrbuch.

\section{Abstract (English) \\ A metasynthesis of textbook analyzes of regional GFL textbooks}

The purpose of the present study is to analyse the primary studies that examined local German textbooks in Turkey and abroad with the Meta-Synthesis Method, and also to show the accumulated knowledge in this field systematically and as a whole. Within the framework of the purpose of the study, the answers are sought for the questions of what the common study fields of the studies that examined the local German textbooks are and which aspects of the books are examined more/less in the primary studies. In the study, it is concluded that most of the primary studies conducted on local German textbooks aim at examining the concept of country knowledge transfer and intercultural learning. It is also concluded that local German textbooks were examined in terms of teaching vocabulary, methodology and project-based learning at the lowest level among the primary studies included in the meta-synthesis.

Keywords: local german course books, course book analysis, meta-synthesis, german as a foreign language, german course books.

Einsendedatum: 20.10 .2020

* Dieser Artikel stellt eine kurze repräsentative Zusammenfassung der von der Autorin vorgelegten Dissertation dar, die von Prof. Dr. Ayten Genç betreut und im Jahre 2019 bei YÖK veröffentlicht wurde. 


\section{EXTENDED ABSTRACT}

It is already known that different German textbook types are used in courses where German is taught as a foreign language. In this context, global textbooks written in Germany, as well as the localized German textbooks developed in these countries for German teaching are used all over the world. This study is about the primary studies that examined local German textbooks, and is based on the idea that textbook analyses are at least as important as the quality of the text books. The literature review shows that there are many scientific studies that examine local German textbooks both in Turkey and abroad, but no studies are detected that systematically bring the results of these studies together. This is the starting point of the present study. The purpose of this study is to examine the findings of primary studies that examine local German textbooks at home and abroad between 1985 and 2019 by using Meta-Synthesis Method. Within the framework of the purpose of the study, the answers are sought to the questions of what the types of the primary studies that examined local German textbooks are, how they show a distribution according to their publications, years, study patterns and samples, what their subject areas are, what the aspects of these books are, which aspects they examine more/less, and which conclusions could be made for the study area according to the results obtained from the synthesis. The Higher Education Council National Thesis Center (YÖK) and TUBITAK ULAKBIM DergiPark databases as well as BASE, MetaGer, Google Academic Search Engines are used in the selection of the studies; and 60 primary studies that are conducted between 1985 and 2019 in Turkey and abroad are included in the meta-synthesis. The studies that are included in the study are analyzed by using Content Analysis Method, and then examined in the context of the purpose of the study, its subject area, method, sampling, data collection tools, and results. As a result of the analysis, 60 primary studies that are included in the metasynthesis reveal that they contain findings for 200 local German textbooks. As a result of the coding processes, the data are listed under 11 themes, and once the data are synthesized among themselves, they are combined with the findings obtained in the evaluations of the textbooks, and the strengths and weaknesses identified for local German textbooks are identified based on the synthesis findings regarding the themes and subthemes. This study is limited to the themes and categories that are included in the author's doctoral thesis, and does not include the synthesis results on the weaknesses and strengths that are identified in local German textbooks. The data obtained as a result of the analysis are interpreted based on the frequency and percentiles, and the results are shown in tables and graphics. It is concluded in study that there are many scientific studies examining local German textbooks between 1985 and 2019, both at home and abroad, the subject is mainly discussed in the master's theses and scientific research papers, a significant part of these studies examined local German textbooks by using various analysis criteria, mixed method studies and studies that employed only quantitative data collection tools are few, and the 60 primary studies included in the meta-synthesis contained the primary findings of 200 local German textbooks. It is also determined that studies conducted on local German textbooks aimed at examining the concept of country knowledge transfer and intercultural learning are more in number, a significant number of studies examine the language used in 
textbooks, and fewer studies focus on grammar transfer, concepts related to language acquisition, visuals and improving language skills in textbooks. In the study, it is also concluded that local German textbooks are examined in terms of linguistic subjects, teaching vocabulary, methodology and project-based learning at the lowest level among the studies included in the meta-synthesis. 


\section{Einleitung}

\section{Regionale Lehrwerke im DaF-Unterricht}

Im Deutsch als Fremdsprache Unterricht wird mit der gemeinsamen Zielsetzung den Lernenden den Zugang zur deutschen Sprache „auf optimalster Weise“ zu gewährleisten, weltweit mit verschiedenen DaF-Lehrwerktypen gearbeitet. In groben Zügen dargestellt, handelt es sich hierbei erstens um regionale DaF-Lehrwerke, die einheimisch vor Ort entwickelt werden, zweitens um überregionale (in der Fachliteratur auch bezeichnet als globale, inter- oder multinationale, universalistische, kommerzielle) DaF-Lehrwerke, die für den internationalen Markt in Deutschland produziert werden und drittens um regionialisierte DaF-Lehrwerke, die meist in Kooperation von ausländischen und einheimischen AutorInnen entstehen (vgl. Tichy 2012: 56).

Ein Blick in die Forschungsliteratur macht ersichtlich, dass gegen die kommerziellen DaF- Lehrwerke eher eine kritische Haltung eingenommen wird, die vor allem damit begründet wird, dass diese für einen großen Markt hergestellt werden und demzufolge in und mit diesen Lehrwerken die regional herrschenden länderspezifischen Unterrichtsbedingungen, Wünsche, Interessen, und Lerntraditionen der Lernenden, aber auch die Unterrichtsziele, -inhalte, und -methoden vor Ort nur wenig berücksichtigt werden. Diese Kritik gegen kommerzielle Lehrwerke und dem damit verbundenen Export von westlichen Bildungszielen und fremden Bildungsskonzepten begann in den Erziehungswissenschaften schon relativ früh: gegen Ende der 60er Jahre. Die Diskussion um die Entwicklung von regionspezifischem und gruppeneigenem Material mit der Zielsetzung den Fremdsprachenunterricht auf die spezifischen Bedürfnisse und Erfahrungen der Lernenden vor Ort anzupassen, wurde im enormen Maße durch die Gedankengänge von Osterloh (1978: 191f.) fortgesetzt, in denen ausdrücklich herausgestellt wird, dass das Ziel nicht mehr eine bloße Darstellung der fremden Wirklichkeit, sondern vielmehr die Entschlüsselung dieser von der eigenen Umwelt der Lernenden aus sein sollte. Demgemäß wird ein an der Umwelt und den praktischen Erfahrungen der Lernenden ausgerichteter Fremdsprachenunterricht befürwortet, damit die erlernte Sprache schließlich zum Instrument der Bewältigung eigener Situationen wird und zur Fortbildung der Identität beiträgt. Mit unterschiedlichen Schwerpunktsetzungen werden Diskussionen und Forderungen nach der Entwicklung von zielgruppenspezifischen Lehr- und Lernmaterialen in den 80er und 90er Jahren fortgesetzt. Die Entscheidungen gegen einen Export von westlichen Bildungskonzepten bringen zugleich eine Ablehnung von eurozentrischen Sichtweisen und veralteten Verfahren im Sprachunterricht mit sich. Auch Gerighausen \& Seel (1982: 23) stellen in diesem Kontext nachdrücklich heraus, dass ,die Ausgansposition wie auch globale Zielvorstellung für regionale Lehrwerke es ist, Bildungsziele und -inhalte, Methoden und Lehr-/Lernformen nicht mehr zu exportieren und anderen Völkern aufzudrängen, sondern auch im Sprachunterricht auf die spezifischen Bedürfnisse und Erfahrungen in der Region Bezug zu nehmen“. In Bezug auf den Einsatz deutscher Lehrmaterialien in Ländern der Dritten Welt fallen auch Schwierigkeiten im Bereich der Kulturspezifika ins Gewicht, da die Unterrichtssprache und das Lernverhalten der Lernenden stark von den Eigenschaften der jeweiligen Kultur geprägt sind (vgl. Krumm 1987: 278). 
Bei der Regionalisierung von DaF-Lehrwerken kann dem Goethe-Institut eine wichtige Rolle beigemessen werden, denn dieses plädierte unter dem Einfluss der kommunikativen Didaktik und des interkulturellen Ansatzes für die Beachtung von kulturspezifischen Eigenschaften der Länder sowie der Lerngewohnheiten in der Region und gründete auf diesem Fundament den Arbeitsbereich „Regionale Lehrwerke“ (vgl. Gerighausen/Seel 1983). Auf dieser Basis erschienen in den 80er Jahren in Zusammenarbeit mit Ländern der dritten Welt Lehrwerke, die eine „regionale Bearbeitung in Deutschland erschienener Lehrwerke“ darstellten (Breitung/Lattaro 2001: 1042). In den folgenden Jahrzehnten führte das gemeinsame Ziel, die spezifischen Bedürfnisse der Lernenden vor Ort näher in Betracht zu ziehen, zu einer weltweiten Entwicklung von länderspezifischen, regionalen DaF-Lehrwerken.

Den Ausgangspunkt dieser Studie stellt die feste Überzeugung dar, dass Lehrwerkanalysen im Kontext regionaler DaF- Lehrwerke ebenso bedeutungsschwer sind, wie die Qualität dieser Lehrwerke selbst. In der Forschungsliteratur lassen sich zahlreiche Studien in Form von Lehrwerkanalysen regionaler DaF-Lehrwerke aufweisen, die die jeweiligen Lehrwerke mit unterschiedlichen Schwerpunktsetzungen und nach den Bewertungsrastern verschiedener Kriterienkataloge analysieren. Die zunehmende Anzahl an Primärstudien, in denen oft verschiedene Schwächen in Bezug auf analysierten Lehrwerke festgestellt werden, die unüberblicklichen oder auch unterschiedlichen Ergebnisse der Primärstudien wurden als Desiderat für eine MetaStudie gedeutet, die die Ergebnisse der einzelnen Arbeiten übersichtlich zusammenfasst und gemeinsam bewertet. Vor diesem Hintergrund beschäftigt sich diese Arbeit mit der Durchführung einer Metasynthese von Primärstudien, die in der Türkei oder im Ausland zwischen den Jahren 1985-2019 verfasst worden sind und regionale DaF-Lehrwerke als Gegenstand in einer Lehrwerkanalyse untersuchen.

\section{Zielsetzung und Relevanz der Untersuchung}

Eine Literaturrecherche nach Primärstudien im Forschungsbereich „Analyse von regionalen DaF-Lehrwerken“" zeigt auf, dass sowohl in der Türkei als auch im Ausland zahlreiche Studien vorliegen, in denen mit verschiedenen Ziel- und Schwerpunktsetzungen regionale DaF-Lehrwerke analysiert werden. Das breite Spektrum an Publikationen belegt, dass der Themenbereich ein beständiges Thema in der Forschungsliteratur ist und seine Aktualität auch bis zum heutigen Zeitpunkt bewahrt. Diese Arbeit wurde im Jahr 2019 als Dissertation vorgelegt und zielt darauf ab, die Ergebnisse von Primärstudien im Bereich „regionale DaF-Lehrwerkanalyse“ übersichtlich als ein Ganzes darzustellen, einen systematischen Vergleich binnen dieser Studien durchzuführen, die Ergebnisse der Einzelstudien zusammenzufassen und auf diesem Wege eine neue und ganzheitliche Darstellung in Hinsicht auf den Forschungsbereich zu schaffen. In der von der Autorin vorgelegten Dissertation wurden die Ergebnisse der Einzelstudien ausführlich dargestellt und auf der Grundlage der Daten Stärken und Schwächen in Bezug auf die analysierten Lehrwerke in den

Primärstudien abgeleitet. Im Zusammenhang dieser Studie wurde das Forschungsvorhaben jedoch mit folgenden Teilfragen begrenzt: 
- Welche Verteilungen sind den im Forschungsbereich verfassten Primärstudien nachzuweisen (Verfassungs- bzw. Publikationsort, -jahr, Art der wissenschaftlichen Studie, Forschungsdesign)

- Welche gemeinsamen Forschungs- und Themenbereiche lassen sich binnen den einzelnen Studien aufweisen?

- Welche Aspekte werden in den Analysen von regionalen DaFLehrwerken häufiger/ weniger häufig thematisiert?

- Welche Schlussfolgerungen lassen sich aus der Synthese für den Forschungsbereich ableiten?

\section{Forschungsdesign}

Die Metasynthese stellt eine Methode dar, die zur „Zusammenführung der Befunde voneinander unabhängigen Primärstudien“ dient (vgl. Sandelowski/Barroso 2003: 907). So zielen Metasynthesen darauf ab, Primärstudien in einem bestimmten Forschungsbereich mit einem qualitativen Verständnis zu untersuchen, Gemeinsamkeiten und Differenzen binnen den Einzelstudien zu bestimmen, zu einem tieferen Einblick in das Forschungsfeld und einer Identifizierung von Prioritäten, besonders für Forscher und Praktiker, die keinen Zugang zu allen verwandten Studien haben, zu verhelfen (vgl. Çalık/Sözbilir 2014: 34). Diese Studie widmet sich der Intention, Primärstudien im Themenbereich „Lehrwerkanalysen von regionalen DaFLehrwerken" die zwischen Jahren 1985-2019 im In- oder Ausland verfasst worden sind, durch qualitative Methoden $\mathrm{zu}$ analysieren und lehnt sich methodisch an die Metasynthese.

\section{Prozess der Datenerhebung und Einschlusskriterien}

Die Datenerhebung für diese Studie wurde in Anlehnung an die Empfehlungen von Noblit \& Hare (1988) geplant und schrittweise durchgeführt. Auf der Basis der Forschungsfragen wurden für das Sampling voraus Kriterien in Bezug auf den Einschluss von Primärstudien definiert. Diesen Kriterien nach umfasst diese Studie sämtliche Primärstudien mit Zugriff auf Volltext, die

- $\quad$ eine Lehrwerkanalyse von regionalen DaF-Lehrwerken darstellen,

- $\quad$ in der Türkei oder im Ausland zwischen den Jahren 1985-2019 in deutscher oder türkischer Sprache abgefasst sind,

- als wissenschaftliche Arbeiten den Genres Master- \& Magisterarbeiten, Dissertationen, Pro-Gradu Arbeiten oder fachliche Zeitschriftenartikel zugeordnet werden können,

- $\quad$ einen standardlichen Wissenschaftlichkeitsgrad erfüllen,

- qualitativer Forschungsart sind oder Mixed-Methods Studien zugeordnet werden können (auch die qualitative Ebene von quantitativen Studien).

In Tab. 1 werden die deutschen und türkischen Schlüsselwörter aufgeführt, anhand deren Literatursuche in den Datenbanken „YÖK Ulusal Tez Merkezi“, „TÜBİTAK 
ULAKBİM DergiPark“ und in den Suchmaschinen „BASE“, „MetaGer“, sowie „Google Scholar“ durchgeführt wurde.

\begin{tabular}{lc}
\hline \multicolumn{1}{c}{ Deutsche Schlüsselwörter } & Türkische Schlüsselwörter \\
\hline regionale DaF-Lehrwerkanalyse & Yerel Almanca ders kitabi incelemesi \\
$\begin{array}{l}\text { DaF-Lehrwerkanalyse } \\
\text { regionale DaF-Lehrwerke }\end{array}$ & Yerel Almanca ders kitabi \\
DaF-Lehrwerk & Almanca ders kitabi incelemesi \\
Lehrwerkanalyse & Almanca ders kitabi \\
Deutschlehrbuch & Yabanc1 dil olarak Almanca \\
Deutsch als Fremdsprache & Almanca \\
Deutsch & \\
\hline
\end{tabular}

Tab. 1: Schlüsselwörter für die Literatursuche

Die Schlüsselwörter „Almanca“ und „Deutsch“ wurden nur in der Datenbank „YÖK Ulusal Tez Merkezi“ verwendet, da die Suche anhand der spezifischeren Schlüsselwörter in dieser Datenbank keine ausreichende Trefferanzahl lieferte.

In Anlehnung an Möhler (2016: 72) wurde die Literatursuche durch andere Recherechetechniken ergänzt (Prüfen von zitierten Arbeiten in verschiedenen Studien, Suche nach Artikeln, die relevante Arbeiten referiert haben, Handsuche in Kongressbänden, Kontaktaufnahme zu ExpertenInnen im Fachbereich). Die Literatursuche in den Dankenbanken und Suchmaschinen lieferte insgesamt 4521, die ergänzende Suche durch Nutzung weiterer Ressorcen ca. 100 Treffer. Nach der Entfernung von Duplikaten und irrelevanten Suchergebnissen, wurden 85 potenzielle Primärstudien auf die Eignung für den Einschluss in die Metasynthese sorgfältig überprüft. Dem Auswahlprozess der identifizierten Primärstudien zufolge, der in Anlehnung an Jones (2007) durchgeführt wurde, wurden in diese Untersuchung insgesamt 60 Primärstudien eingeschlossen.

\section{Der Kodierungsprozess}

Vorweg wurde ein Datenentnahmeraster vorbereitet, auf dem nach einer detaillierten Lektüre einer jeden Primärstudie, die Informationen zu den betreffenden Teilen der Originalarbeiten festgehalten wurden. Nach einer Untersuchung der eingeschlossenen Primärstudien auf ihre Zielsetzungen hin, wurden unter Beachtung der Fachliteratur für jeden Themenbereich entsprechende Codes erschaffen. Zudem wurden den Originalarbeiten, um Vergleiche und eine systematische Bewertung der Primärstudien zu erleichtern, Studiencodes in Form von A1, A2, A3, ... A60 verliehen.

\section{Maßnahmen zur Validität und Reliabilität}

Die interne Validität wurde unter anderem dadurch sichergestellt, dass die Recherche im Bereich der Fachliteratur und in Bezug auf die Erhebung von Daten den gesamten 
Forschungsprozess begleitete. Da aufgrund des Forschungsdesigns eine Variation der Datenerhebungsinstrumente nicht möglich war, wurde in dieser Arbeit eine Triangulation in dem Sinne durchgeführt, dass verschiedene Arten von Primärstudien (siehe Tab. 2) in die Metasynthese eingeschlossen wurden. Um ein möglichst repräsentatives Bild zu schaffen, wurde darauf abgezielt, möglichst viele Primärstudien, die die Einschlusskriterien der Synthese erfüllen, in die Studie einzuschließen. Durch eine ausführliche Formulierung und detaillierte Darstellung der Forschungsergebnisse, wurde die externe Validität der Arbeit gewährleistet. Sämtliche Rohdaten, Codierungen bei der Analyse der Daten, Arbeitsschritte und -prozesse sowie Erkenntnisse zur Berichterstattung wurden sorgfältig aufbewahrt. Da die Daten in dieser Arbeit durch eine Inhaltsanalyse untersucht wurden, wurden einerseits Maßnahmen zur Interrater Reliabilität ergriffen, andererseits wurden die Kodierungen nach einem Zeitraum von einem Monat wiederholt durchgeführt. Jeweils drei von den eingeschlossenen Studien wurden von zwei voneinander unabhängigen Fachexperten anhand des Datenentnahmerasters durchgearbeitet. Der Vergleich der Ergebnisse anhand der Formel von Krippendorf (2011) zeigte mit einer Zahl von 0.90, dass die Kodierungen konsequent durchgeführt wurden. Die Codes, bei denen kein Konsens herrschte, wurden überarbeitet und korrigiert. Eine erneute Kodierung nach einem Zeitraum von einem Monat zeigte auf, dass sich die Ergebnisse mit einer Zahl von 0.97 deckten.

\section{Datenanalyse}

Zur Aggregation der Rohdaten wurde ein Datenentnahmeraster verwendet, das primär für die Einzelanalyse einer jeden eingeschlossenen Primärstudie diente. Die Objekte des Aggregationsverfahrens standen in engem Bezug zu den Forschungsfragen dieser Studie, demnach wurden bei den Einzelanalysen detaillierte Informationen in Bezug auf die Ziele, die verwendeten Methoden, die Befunde und Ergebnisse aus den Lehrwerkanalysen entnommen und in den Datenentnahmerastern ausführlich dokumentiert. Die aus den Einzelanalysen entnommenen Daten wurden nach der von Miles \& Hubermann (1994) vorgeschlagenen cross-case Technik analysiert. Da bei diesem Prozess auch die Kriterien zur Begutachtung von DaF-Lehrwerken aus verschiedenen Kriterienkatalogen beachtet (vordefinierte Codes), diesen aber auch neue Codes hinzugefügt wurden, wurden in dieser Studie sowohl induktive als auch deduktive Verfahren verwendet.

\section{Befunde}

In diesem Abschnitt werden die Befunde dargestellt, die anhand den insgesamt eingeschlossenen 60 Primärstudien in der Metasynthese erhoben wurden. 


\section{Verteilungen der Primärstudien nach Art und Verfassungs- bzw. Publikationsort}

Tab. 2 verdeutlicht die Arten der eingeschlossenen Primärstudien. Die Prozentzahlen geben Aufschluss darüber, welchen Anteil die jeweilige Kategorie in Bezug auf die insgesamt eingeschlossenen Inland- bzw. Auslandsstudien einnimmt.

\begin{tabular}{|c|c|c|c|c|c|c|}
\hline Art der Studie & $\begin{array}{c}\text { Anzahl der } \\
\text { Inlandstudien } \\
\text { (f) }\end{array}$ & $(\%)$ & $\begin{array}{c}\text { Anzahl der } \\
\text { Auslandsstudien } \\
\text { (f) }\end{array}$ & $(\%)$ & $\begin{array}{c}\text { Insgesamt } \\
\text { (f) }\end{array}$ & $(\%)$ \\
\hline Master- \& Magisterarbeiten & 13 & 48.14 & 11 & 33.33 & 24 & 40 \\
\hline Zeitschriftenartikel & 10 & 37.03 & 14 & 42.42 & 24 & 40 \\
\hline Dissertationen & 4 & 14.81 & 2 & 6.06 & 6 & 10 \\
\hline Pro-Gradu Arbeiten & 0 & 0 & 6 & 16.18 & 6 & 10 \\
\hline Insgesamt & 27 & 100 & 33 & 100 & 60 & 100 \\
\hline
\end{tabular}

Tab. 2: Verteilungen der Primärstudien nach ihrer Art

Wie aus Tab. 2 ersichtlich, machen von den in die Metasynthese eingeschlossenen Primärstudien Master- \& Magisterarbeiten und wissenschaftliche Zeitschriftenartikel die höchsten Anteile aus. Den zweiten Platz nehmen die inkludierten Dissertationen und Pro-Gradu Arbeiten ein. Von den insgesamt eingeschlossenen Primärstudien stammen 27 aus der Türkei und 33 aus dem Ausland. Bezüglich der Primärstudien aus der Türkei entfällt der größte Anteil der Studien auf die Master- \& Magisterarbeiten. Den zweiten Platz belegen die eingeschlossenen Zeitschriftenartikel. Ferner wurden 4 Dissertationen aus der Türkei eingeschlossen, die im Bereich der Inlandstudien den letzten Platz einnehmen. In Bezug auf die Auslandsstudien entfällt der größte Anteil auf die Kategorie Zeitschriftenartikel. Den zweiten Platz belegt die Kategorie Master- \& Magisterarbeiten, die geringsten Anteile verzeichnen in diesem Bereich jedoch die eingeschlossenen Dissertationen und Pro-Gradu Arbeiten. Tab. 3 gibt Auskunft über die Anzahl der eingeschlossenen Primärstudien aus den jeweiligen Ländern.

\begin{tabular}{lcc}
\hline Land & $\begin{array}{c}\text { Anzahl der Studien } \\
\text { (f) }\end{array}$ & $\mathbf{( \% )}$ \\
\hline Türkei & 27 & 45 \\
Finnland & 14 & 23.33 \\
Deutschland & 9 & 15 \\
\hline Japan & 2 & 3.33 \\
Norwegen & 2 & \\
Schweiz & 2 & 1.66 \\
\hline Kroatien & 1 & \\
Polen & 1 & $\mathbf{1 0 0}$ \\
Niederlande & 1 & \\
Brasilien & 1 & $\mathbf{6 0}$ \\
Insgesamt & \multicolumn{2}{c}{} \\
\hline Tab. 3: Verteilungen der Primärstudien nach ihrem Verfassungs- bzw. Publikationsort
\end{tabular}


Tab. 3 zeigt, dass 27 Studien aus der Türkei stammen und die die Primärstudien aus Finnland den höchsten Anteil im Bereich der Auslandsstudien ausmachen. 9 Primärstudien stammen aus Deutschland, auf die Länder Japan, Norwegen und die Schweiz entfallen je 2 Primärstudien. Studien aus Ländern wie Kroatien, Polen, die Niederlande und Brasilien sind mit je einer Primärstudie deutlich niedriger vertreten.

\section{Verteilungen der Primärstudien nach ihrem Verfassungs- bzw. Publikationjahr}

Nach dem in den Einschlusskriterien festgelegten Zeitraum umfasst diese Metasynthese Primärstudien, die zwischen den Jahren 1985-2019 verfasst worden sind. Abb. 3 verdeutlicht die Anzahl der Primärstudien, die im festgelegten Zeitraum identifiziert werden konnten und in die Metasynthese eingeschlossen wurden.

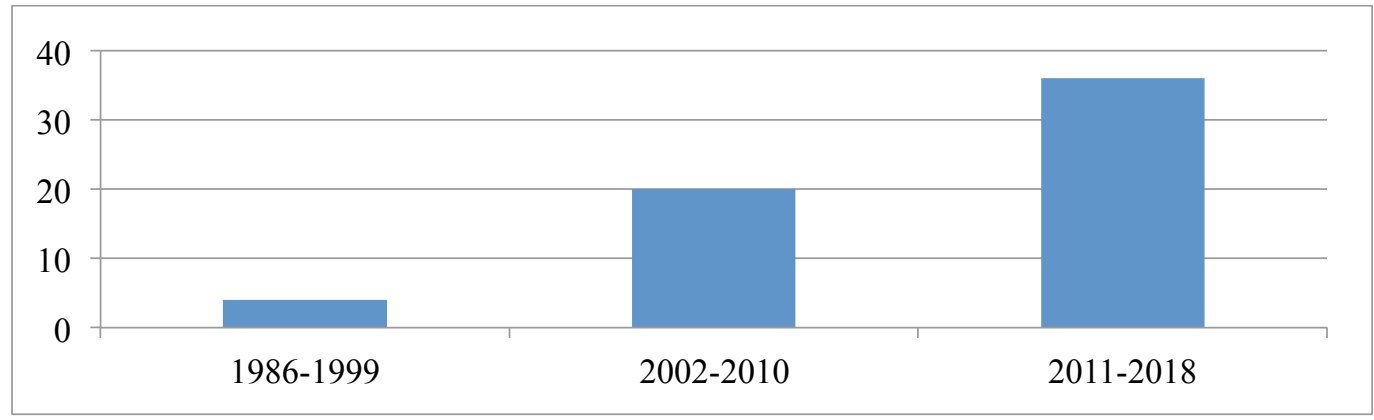

Abb. 1: Verteilungen der Primärstudien nach ihrem Verfassungs- bzw. Publikationsjahr

Abb. 1 verdeutlicht, dass der in den Einschlusskriterien festgelegte Zeitraum mit den eingeschlossenen Primärstudien gut vertreten ist, aus den Jahren 1985 und 2019 keine Primärstudien identifiziert werden konnten, die die Einschlusskriterien dieser Metasynthese erfüllen und die Zahl an wissenschaftlichen Studien im Rahmen regionaler DaF-Lehrwerke im Laufe der Zeit zugenommen hat. Mit 36 Originalarbeiten wurden überwiegend gegenwartsnahe Primärstudien (2011-2018) in die Studie eingeschlossen. Ferner wurden 20 Primärstudien, die zwischen den Jahren 2002-2010 und 4 Primärstudien in die Synthese inkludiert, die zwischen dem Zeitraum von 19861999 verfasst worden sind.

\section{Verteilungen der Primärstudien nach ihrem Forschungsdesign}

Tab. 4 gibt Aufschluss über die verwendeten Methoden in den insgesamt eingeschlossenen 60 Primärstudien.

\begin{tabular}{lcc}
\hline Forschungsdesign & (f) & $(\mathbf{\%})$ \\
\hline Qualitative Methoden & 51 & 85 \\
Mixed-Methods & 5 & 8.33 \\
Quantitative Methoden & 4 & 6.66 \\
Insgesamt & $\mathbf{6 0}$ & $\mathbf{1 0 0}$ \\
\hline
\end{tabular}

Tab. 4: Verteilungen der Primärstudien nach ihrem Forschungsdesign 
Tab. 4 verdeutlicht, dass 51 Primärstudien (85\%) mit deskriptivem Forschungsdesign den höchsten Anteil verzeichnen. Mixed-Methods und Studien, bei denen Fragtechniken zur Ermittlung bestimmer Eigenschaften mit Lernenden oder Lehrenden verwendet werden, machen deutlich den geringsten Anteil aus.

\section{Verteilungen der Primärstudien nach ihren Themenbereichen}

Die eingeschlossenen Primärstudien wurden in Entsprechung zu ihren Themen, ihren Zielsetzungen und Ergebnissen nach in deskriptiven Themenbereichen geordnet. In Tab. 5 werden die insgesamt 11 ihrer Rangfolge nach geordneten Themenbereiche aufgeführt.

\begin{tabular}{llcc}
\hline Themenbereiche & Primärstudien & (f) & $(\mathbf{\%})$ \\
\hline $\begin{array}{l}\text { Landeskunde \& } \\
\text { interkulturelles Lernen }\end{array}$ & A12, A13, A14, A15, A17, A21, A29, A31, A32, & 17 & 28.33 \\
\hline Multidimensionale Analysen & A36, A40, A41, A42, A47, A49, A50, A52 & & 13.33 \\
Sprache & A1, A6, A7, A37, A53, A55, A56, A57 & 8 & 13.33 \\
\hline Grammatik & A4, A5, A16, A22, A23, A33, A34, A46 & 8 & 10 \\
Konzepte des Spracherwerbs & A24, A27, A35, A45, A51, A54 & 6 & 10 \\
\hline Visualität & A9, A10, A25, A30, A39, A59 & 6 & 8.33 \\
Sprachliche Fertigkeiten & A3, A11, A38, A44, A48 & 5 & 8.33 \\
\hline Linguistische Gegenstände & A8, A18, A20, A28, A58 & 5 & 3.33 \\
\hline Wortschatz & A19, A60 & 2 & 1.66 \\
Vermittlungskonzepte & A2 & 1 & 1.66 \\
Selbstorganisationsformen & A26 & 1 & 1.66 \\
\hline Insgesamt & & 1 & $\mathbf{1 0 0}$ \\
\hline
\end{tabular}

Tab. 5: Verteilungen der Primärstudien nach ihren Themenbereichen

Tab. 5 zeigt, dass der Themenbereich „Landeskunde und interkulturelles Lernen“ deutlich den höchsten Anteil an Studien verzeichnet. Die Primärstudien, die auf eine ganzheitliche Analyse von Lehrwerken abzielen und keinen spezifischen Aspekt im Fokus ihrer Lehrwerkanalyse haben, wurden dem Themenbereich „Multidimensionale Lehrwerkanalysen“ zugeordnet und machen einen Anteil von $13.33 \%$ aus. Der Themenbereich „Sprache“ ist desgleichen mit 8 Primärstudien belegt. Diesem folgen die Primärstudien der zwei Themenbereiche „Grammatik“ und „Konzepte des Spracherwerbs“ mit jeweils 6 Studien. Die Themenbereiche „Visualität" und „Sprachliche Fertigkeiten“ sind mit jeweils 5 Studien geringer vertreten. Deutlich weniger Studien werden dem Themenbereich „Linguistische Gegenstände“ zugeordnet, jedoch verzeichnen die Bereiche „Wortschatz“, „Vermittlungskonzepte“ und „Selbstorganisationsformen“ die geringsten Anteile.

\section{Kategorien zu den Themenbereichen}

Unter Beachtung der einzelnen methodischen Schritte für Metasynthesen wurden die eingeschlossenen Primärstudien sorgfältig analysiert. Diesem Prozess zufolge wurden 
$\mathrm{zu}$ den einzelnen Themenbereichen unter bestimmen Kategorien Schlüsselwörter und Begriffe generiert, die die Gemeinsamkeiten und Unterschiede binnen den Studien verdeutlichen sollen.

Tab. 6 gibt Auskunft über folgende Aspekte: In der linken Spalte werden die Themenbereiche und in der mittleren Spalte die einzelnen Kategorien zu den Themenbereichen aufgeführt. Die rechten Spalten informieren über die Anzahl (und Prozentzahl) der Studien im Themenbereich, in der die jeweilige Kategorie behandelt wird.

\begin{tabular}{|c|c|c|c|}
\hline Themenbereiche & Kategorien & $\mathbf{f}$ & $(\%)$ \\
\hline \multirow{15}{*}{$\begin{array}{l}\text { Landeskunde \& } \\
\text { interkulturelles Lernen }\end{array}$} & $\begin{array}{l}\text { 1. Texte \& Themen } \\
\text { 2. Interkulturalität }\end{array}$ & $\begin{array}{l}11 \\
11\end{array}$ & 64.70 \\
\hline & 3. Einbezug aller deutschsprachigen Länder & 10 & 58.82 \\
\hline & 4. Einbezug von literarischen Texten & 8 & 47.05 \\
\hline & 5. Sprachliche Diversität & 7 & \multirow{3}{*}{41.17} \\
\hline & 6. Subjekte im Lehrwerk & 7 & \\
\hline & 7. Darstellung von Kultur & 7 & \\
\hline & 8. Vorurteile, Stereotype und Klischees & 6 & 35.29 \\
\hline & $\begin{array}{l}\text { 9. Einsatz von Karten und Fotos zur Darstellung } \\
\text { von Geographie }\end{array}$ & 5 & 29.41 \\
\hline & 10. Darstellung der Gesellschaft & 4 & \multirow{3}{*}{23.52} \\
\hline & 11. Vorhandensein von Zusatzmaterial & 4 & \\
\hline & $\begin{array}{l}\text { 12. Aufgabenarten zur kritischen } \\
\text { Auseinandersetzung mit Inhalten }\end{array}$ & 4 & \\
\hline & $\begin{array}{l}\text { 13. Übereinstimmung der landeskundlichen Inhalte } \\
\text { mit den Vorschriften des Lehrplans }\end{array}$ & 3 & \multirow{4}{*}{17.64} \\
\hline & 14. Authentizität von Bildern & 3 & \\
\hline & 15. Behandlung von historischen Ereignissen & 3 & \\
\hline & 16. Vielseitige Behandlung des Alltagslebens & 3 & \\
\hline \multirow{7}{*}{$\begin{array}{l}\text { Multidimensionale } \\
\text { Analysen }\end{array}$} & 1. Sprache & 6 & \multirow{2}{*}{75} \\
\hline & 2. Sprachliche Fertigkeiten & 6 & \\
\hline & 3. Landeskunde \& interkulturelles Lernen & 5 & \multirow{2}{*}{62.50} \\
\hline & 4. Grammatik & 5 & \\
\hline & 5. Einsatz von interaktiven Sozialformen & 4 & 50 \\
\hline & 6. Förderung von Lernerautonomie & 3 & 37.50 \\
\hline & 7. Visualität & 2 & 25 \\
\hline \multirow{8}{*}{ Sprache } & 1. Texte & 7 & \multirow{2}{*}{87.50} \\
\hline & 2. Authentizität der Sprache & 7 & \\
\hline & 3. Förderung der kommunikativen Kompetenz & 4 & 50 \\
\hline & 4. Spektrum an Beispielen für sprachliche Vielfalt & 3 & \multirow{3}{*}{37.50} \\
\hline & 5. Orientierung an der Standardsprache & 3 & \\
\hline & 6. Einsatz von regionalen Varietäten & 3 & \\
\hline & $\begin{array}{l}\text { 7. Berücksichtigung von kulturell- sprachlichen } \\
\text { Unterschieden }\end{array}$ & 2 & 25 \\
\hline & 8. Aussprache und Intonation & 1 & 12.50 \\
\hline \multirow{5}{*}{ Grammatik } & 1. Grammatikübungen & 6 & \multirow{2}{*}{100} \\
\hline & 2. Grammatikdarstellung & 6 & \\
\hline & 3. Grammatikkonzept & 5 & 83.33 \\
\hline & 4. Grammatikprogression & 4 & \\
\hline & $\begin{array}{l}\text { 5. Grammatikbezogene Hinweise im } \\
\text { Inhaltsverzeichnis }\end{array}$ & 4 & 66.66 \\
\hline
\end{tabular}




\begin{tabular}{|c|c|c|c|}
\hline & 6. Förderung von Sprachbewusstheit & 2 & 33.33 \\
\hline Konzepte des & 1. Sprache der Sprachvergleiche & 6 & 100 \\
\hline \multirow{11}{*}{ Spracherwerbs } & 2. Bereich der Sprachvergleiche & 4 & 66.66 \\
\hline & 3. Förderung von Sprachbewusstheit & 3 & \multirow{4}{*}{50} \\
\hline & 4. Behandlung von möglichen Interferenzfehlern & 3 & \\
\hline & 5. Explizite Behandlung von Lernstrategien & 3 & \\
\hline & 6. Typen der Lernstrategien & 3 & \\
\hline & $\begin{array}{l}\text { 7. Kontinuierlicher Einbezug von tertiärsprachlich- } \\
\text { en Prinzipien }\end{array}$ & 2 & \multirow{6}{*}{33.33} \\
\hline & 8. Explizite Thematisierung von Möglichkeiten aus & 2 & \\
\hline & Transfer \& Sprachvergleich & & \\
\hline & 9. Behandlung von interkulturellen Erfahrungen & 2 & \\
\hline & 10. Einsatz von Internationalismen & 2 & \\
\hline & 11. Einsatz von Anglizismen & 2 & \\
\hline \multirow{8}{*}{ Visualität } & 1. Bildfunktionen & 5 & \multirow{3}{*}{100} \\
\hline & 2. Technische Qualität & 5 & \\
\hline & $\begin{array}{l}\text { 3. Vergleich mit dem pädagogischen Konzept der } \\
\text { Ausgangskultur }\end{array}$ & 5 & \\
\hline & 4. Bildsorten & 4 & 80 \\
\hline & 5. Mitteilungsabsicht der visuellen Komponente & 3 & \\
\hline & $\begin{array}{l}\text { 6. Hinweise zur Bildverwendung im } \\
\text { Lehrerhandbuch }\end{array}$ & 3 & 60 \\
\hline & $\begin{array}{l}\text { 7. Angemessene Gestaltung makrotypografischer } \\
\text { Komponente }\end{array}$ & 2 & 40 \\
\hline & 8. Kreative Bildarbeit & 1 & 20 \\
\hline \multirow{5}{*}{$\begin{array}{l}\text { Sprachliche } \\
\text { Fertigkeiten }\end{array}$} & 1. Sprechfertigkeit & 5 & 100 \\
\hline & 2. Leseverstehen & 3 & \multirow{2}{*}{60} \\
\hline & 3. Hörverstehen & 3 & \\
\hline & 4. Schreibfertigkeit & 1 & \multirow[t]{2}{*}{20} \\
\hline & $\begin{array}{l}\text { 5. Ausgewogene Behandlung der sprachlichen } \\
\text { Fertigkeiten }\end{array}$ & 1 & \\
\hline Linguistische & 1. Einsatz von Idiomen & 1 & 50 \\
\hline Gegenstände & 2. Modifikationen von Sprichwörtern & 1 & 50 \\
\hline Wortschatz & 1. Wortschatzvermittlung und -darstellung & 1 & 100 \\
\hline $\begin{array}{l}\text { Vermittlungs- } \\
\text { konzepte }\end{array}$ & $\begin{array}{l}\text { 1. Übereinstimmung mit den Merkmalen der } \\
\text { Vermittlungsmethoden }\end{array}$ & 1 & 100 \\
\hline Selbstorganisationsformen & 1. Projektorientierung & 1 & 100 \\
\hline
\end{tabular}

Tab. 6 verdeutlicht zum einen die Forschungs- und Themenbereiche und zum anderen die Kategorien, die in den analysierten Primärstudien gemeinsam behandelt werden. In Zusammenhang der Forschungsfragen dieser Arbeit läßt sich feststellen, dass sich im Themenbereich „Landeskunde und interkulturelles Lernen“ 16 gemeinsame Kategorien ergeben, auf die die AutorInnen in den Primärstudien Bezug nehmen. In den Primärstudien dieses Themenbereichs werden am häufigsten (in 11 von 17 Primärstudien) die beiden Kategorien „Texte \& Themen“ und „Interkulturalität“ behandelt. Unter der Kategorie „Texte \& Themen“ wird in den Primärstudien des näheren der Frage nachgegangen, ob die Texte und Themen in den analysierten regionalen DaF-Lehrwerken authentisch, altersgerecht, relevant, aktuell und abwechslungsreich gestaltet sind. Weiterhin werden unter dieser Kategorie die Aspekte 
behandelt, ob die Texte und Themen progressionell zugeordnet sind und Vorkenntnisse und Erwartungen der Zielgruppe in den analysierten Lehrwerken berücksichtigt werden. Unter der Kategorie „Interkulturalität“ stehen Fragen wie die Berücksichtigung von kulturkontrastiven Aspekten, die Behandlung von Beziehungen zwischen Ausgangsund Zielkultur sowie die die Thematisierung von anderen Ländern in den analysierten Lehrwerken im Mittelpunkt. Auch der Einbezug von allen deutschsprachigen Ländern und die Verfolgung des DACH- Prinzips werden in über der Hälfte der Primärstudien erforscht. Fast in der Hälfte der Primärstudien (47.05 \%) wird untersucht, ob oder inwieweit literarische Texte in die analysierten Lehrwerke einbezogen werden. Relativ häufig werden in den Primärstudien dieses Themenbereichs (41.17 \%) die sprachliche Diversität, die Subjekte und die Darstellung von Kultur in den Lehrwerken untersucht. Bei der Kategorie ,sprachliche Diversität“, wird im näheren den Fragen nachgegangen, ob in den Lehrwerken die Plurizentrik des Deutschen mit seinen Varietäten thematisiert wird und ob auch weitere sprachliche Variationsdimensionen wie Jugendsprache und Umgangssprache eingesetzt werden. Die Kategorie „Subjekte im Lehrwerk“ konzentriert sich auf die Untersuchung der Identifikationsmöglichkeiten mit den Personen in den Lehrwerken, die Behandlung von Charakteristika der Deutschen und die Frage nach dem Vorhandensein von geschlechtsspezifischen Stereotypen in den Lehrwerken. Die Kategorie „Darstellung der Kultur“ widmet sich vor allem der Frage, inwieweit die Themen Kunst, Theater, Film und Kino in die Lehrwerke einbezogen werden, aber auch den Fragestellungen, inwieweit Sitten, Gebräuche und Feste der Zielkultur vorgestellt werden. Weiterhin werden die analysierten Lehrwerke unter dieser Kategorie daraufhin untersucht, ob Tabuthemen und religiöse Erfahrungen in den Lehrwerken behandelt werden.

Ferner lässt sich aus den Daten in Tab. 6 ableiten, dass in den Primärstudien des zweiten Themenbereichs „Multidimensionale Lehrwerkanalysen“ am häufigsten die zwei Kategorien „Sprache“ und „Sprachliche Fertigkeiten“ (in 75 \% der Studien dieses Themenbereichs) untersucht werden. Unter der Kategorie „Sprache“ kommen vor allem die Eigenschaften der in den Lehrwerken verwendeten Texte zum Ausdruck. Im näheren geht es hier um die Authentizität der Texte, die Vielfalt der Textsorten, die Angemessenheit der Texte an die Zielgruppe sowie um die Situativität und kontextuelle Einbettung der Texte. Dazu werden aber auch weitere Aspekte behandelt, wie die Fragestellung, ob sich die Lehrwerke überwiegend an der Standardsprache des Deutschen orientieren oder ob auch Beispiele zur sprachlichen Vielfalt (Jugend- $u$. Umgangssprache) in den Lehrwerken zu finden sind. Überdies aber auch Aspekte zur Aussprache und Intonation (systematische Behandlung, Berücksichtigung von kontrastiv-schwierigen Lauten) und zum Hörmaterial (Vielfalt, Authentizität), das in den Lehrwerken verwendet wird. Unter der Kategorie „Sprachliche Fertigkeiten“ konzentrieren sich die AutorInnen der Primärstudien überwiegend darauf, ob in den analysierten Lehrwerken die einzelnen sprachlichen Fertigkeiten ausgewogen behandelt werden.

Den zweiten Platz nehmen in diesem Themenbereich die zwei Kategorien „Landeskunde \& interkulturelles Lernen“ und „Grammatik“ ein, die in $62.50 \%$ der 
Primärstudien behandelt werden. Hier kommen Fragestellungen wie die Berücksichtigung von kulturkontrastiven Aspekten und die Vermittlung eines realtischen Deutschlandbildes zum Vorschein. Die Kategorie „Grammatik“ konzentriert sich überwiegend auf die Grammatikprogression, -darstellung, -übungen und das Grammatikonzept der Lehrwerke. Weiterhin lässt sich feststellen, dass in der Hälfte der Studien dieses Themenbereichs erforscht wird, inwieweit die Lehrwerke den Einsatz von interaktiven Sozialformen fördern.

Im Mittelpunkt der Primärstudien des dritten Themenbereichs „Sprache“ stehen die zwei Kategorien „Texte“ und „Authentizität der Sprache“, die beide in $87.50 \%$ der Studien behandelt werden. Unter der Kategorie „Texte“ wird hierbei in den Primärstudien überwiegend untersucht, ob und inwiefern die Texte in den Lehrwerken authentisch sind. Weiterhin lässt sich aus den Daten in Tab. 6 ableiten, dass in der Hälfte der Primärstudien dieses Themenbereichs der Frage nachgegangen wird, inwieweit die analysierten Lehrwerke die kommunikative Kompetenz der Lernenden fördern.

In Bezug auf den vierten Themenbereich „Grammatik“ lässt sich aus den Daten ableiten, dass am häufigsten die Kategorie „Grammatikübungen“ und „Grammatikdarstellung“ behandelt werden. Unter der Kategorie „Grammatikübungen“ kommt hier vor allem die Frage nach der Kontextualität der Übungen und die Sprache der Arbeitsanweisungen zum Vorschein. Bei der Kategorie „Grammatikdarstellung“ konzentrieren sich die AutorInnen überwiegend auf die Regelgebung (Einsatz von Muttersprache, Vorhandensein von Hinweisen und Erklärungen) in den Lehrwerken. Weiterhin fällt auf, dass das Grammatikkonzept in den Primärstudien dieses Themenbereichs sehr häufig analysiert (83. $33 \%$ der Studien) wird. Über die Hälfte der Studien (66.66 \%) nehmen in der Lehrwerkanalyse Bezug auf die Grammatikprogression und auf die Frage, ob im Inhaltsverzeichnis des Lehrwerks grammatikbezogene Hinweise vorhanden sind.

In allen eingeschlossenen Primärstudien des fünften Themenbereichs „Konzepte des Spracherwerbs“" wird die Sprache, in der Sprachvergleiche in den Lehrwerken vorgenommen werden (Sprachvergleiche mit der L1, mit dem Englischen als L2 oder mit anderen Sprachen) thematisiert. In mehr als der Hälfte (66.66 \%) der Studien wird auch auf den Bereich (Wortschatz, Grammatik, Aussprache, Übersetzungen), in dem die Sprachvergleiche durchgeführt werden, Bezug genommen. Weiterhin lässt sich aus den Daten entnehmen, dass in der Hälfte der Studien untersucht wird, inwiefern die Lehrwerke die Sprachbewusstheit der Lernenden fördern, ob auf mögliche Interferenzfehler Bezug genommen wird, welche Lernstrategien vermittelt werden und ob eine explizite Thematisierung von Lernstrategien vorgenommen wird.

Die Funktion der in den Lehrwerken verwendeten Bilder und deren technische Qualität werden in allen eingeschlossenen Primärstudien des sechsten Themenbereichs „Visualität“ thematisiert. Auch werden in allen Studien Vergleiche mit dem pädagogischen Konzept der Ausgangskultur (Angemessenheit der Bilder an die Altersgruppe, Tabuthemen, Berücksichtigung von Vorkenntnissen der Zielgruppe) 
vorgenommen. Sehr häufig (in $80 \%$ der Studien) werden auf in den Lehrwerken verwendeten Bildsorten Bezug genommen. Die Mitteilungsabsicht der visuellen Komponente (Klarheit des situativen Rahmens, Deutlichkeit der kommunikativen Absicht der Personen, Erkennbarkeit von Rollen der Personen) und ob Hinweise zur Bildverwendung in den Lehrerhandbüchern vorhanden sind, sind Aspekte, die in mehr als der Hälfte $(60 \%)$ der Studien behandelt werden.

Während die Sprechfertigkeit in allen Primärstudien des siebten Themenbereichs „Sprachliche Fertigkeiten“ behandelt wird, wird auf das Lese- und Hörverstehen in nur ein wenig über der Hälfte (60\%) der Studien Bezug genommen.

Der achte Themenbereich „Linguistische Gegenstände“ umfasst zwei Primärstudien, die sich in ihrem Forschungsvorhaben zum einen mit dem Einsatz von Idiomen (Anzahl der Idiome und Behandlung) und zum anderen mit Modifikationen von Sprichwörtern (Anzahl und Modifikationsarten und deren Funktionen) in regionalen DaF-Lehrwerken befassen. Die letzten drei Themenbereiche „Wortschatz“, „Vermitlungskonzepte“ und „Selbstorganisationsformen“ schließen jeweils eine Primärstudie ein. Die Studie im Themenbereich „Wortschatz“ konzentriert sich darauf, ob in den analysierten Lehrwerken Wortbildungsregeln systematisch eingeführt werden, die Wortschatzauswahl begründet wird, auf den Einsatz von visuellen und verbalen Hilfsmitteln bei der Wortschatzpräsentation, die Übungsformen, die Förderung von Lernerautonomie sowie auf die Vermittlung von Lernstrategien. Während in den Lehrwerkanalysen des Themenbereichs „Vermittlungskonzepte“ im Mittelpunkt die Übereinstimmung mit den Merkmalen der verschiedenen Vermittlungskonzepte liegen, werden in den Primärstudien des letzten Themenbereichs „Selbstorganisationsformen“ unter der Kategorie „Projektorientierung“ die Lehrwerke daraufhin untersucht, ob und inwieweit in den Lehrwerken Projektaufgaben eingesetzt werden und diese Möglichkeiten zur Sozialisierung der Lernenden ermöglichen.

\section{Schlussbetrachtung}

Aus der Analyse der in diese Metasynthese eingeschlossenen Primärstudien lässt sich schließen, dass sowohl im In- als auch im Ausland zahlreiche Lehrwerkanalysen von regionalen DaF-Lehrwerken aufzufinden sind und der Themenbereich häufig in fachwissenschaftlichen Artikeln sowie Master- \& Magisterarbeiten, in Dissertationen dagegen seltener behandelt wird. In Bezug auf den in den Einschlusskriterien festgelegten Zeitraum von 1985- 2019 lässt sich festhalten, dass in der Metasynthese überwiegend gegenwartsnahe Studien eingeschlossen und analysiert wurden. Aus den Verteilungen der in den Primärstudien verwendeten Methoden lässt sich schlussfolgern, dass Studien qualitativer Forschungsart deutlich die höchsten Anteile verzeichnen. Mixed-Methods Studien und Studien, bei denen Fragetechniken zur Ermittlung bestimmer Eigenschaften von Lehrwerken mit Lernenden oder Lehrenden verwendet werden, machen deutlich die geringsten Anteile in der Metasynthese aus. In dieser Hinsicht würden, wie auch in der Forschungsliteratur darauf hingewiesen, weitere Studien, die über werkanalytische Vorgehensweisen herausgehen, nähere Anhaltspunkte 
über die Wirkung von Lehrwerken auf konkreten Lerngruppen liefern, genauere Anstöße zu den Bedürfnissen, Erwartungen und Wünschen der Lernenden beim Erlernen des Deutschen als Fremdsprache geben und somit $\mathrm{zu}$ einer genaueren Anpassung der Lehrwerke an die Zielgruppen vor Ort verhelfen (vgl. Rösler/Schart 2016: 490).

Die Ergebnisse der Synthese verdeutlichen, dass die in den Primärstudien untersuchten regionalen DaF-Lehrwerke sehr häufig in Bezug auf die Vermittlung von landeskundlichen Gegenständen und in diesem Zusammenhang auf den Aspekt von Interkulturalität und die Eigenschaften der in den Lehrwerkwerken enthaltenen Texte und Themen analysiert werden. Weniger untersucht wird in diesem Kontext, ob Karten und Fotos zur Darstellung der Geographie in den Lehrwerken zielgerecht eingesetzt werden, wie die Gesellschaft dargestellt wird, ob Zusatzmaterial zu dem Bereich Landeskunde vorhanden ist und ob in den Lehrwerken Aufgabenarten zu finden sind, die eine kritische Auseinandersetzung mit Lerninhalten fördern. Viel weniger wird in den Lehrwerkanalysen dieses Themenbereiches darauf Bezug genommen, ob die Lehrwerkinhalte mit den Vorschriften des Lehrplans übereinstimmen und wie authentisch die verwendeten die Bilder in den Lehrwerken sind. In diesem Sinne ist für weitere Studien im Forschungsfeld darauf anzuregen, die Authentizität der in den Lehrwerken verwendeten Bilder näher zu erforschen, um somit dem Anspruch der Realitätsnähe mit der Untermauerung von authentischen Materialien gerecht zu werden (vgl. Demiryay 2016: 240).

Weiterhin zeigen die Ergebnisse, dass nach dem Themenbereich „Landeskunde und interkulturelles Lernen“ regionale DaF-Lehrwerke häufig als Ganzes und mit Fokus auf verschiedene Teilaspekte untersucht werden. In den Lehrwerkanalysen dieses Themenbereichs fallen Aspekte wie die Eigenschaft der Sprache und die Entwicklung der sprachlichen Fertigkeiten in den Schwerpunkt. Eine weitere Implikation betrifft in diesem Rahmen die deutlich weniger behandelten Fragen in den Lehrwerkanalysen, inwieweit interaktive Sozialformen eingesetzt werden, die Lernerautonomie gefördert wird und die Visualität der Lehrwerke für die Zielgruppe geeignet ist.

Ferner geht aus den Ergebnissen der Synthese hervor, dass Lehrwerkanalysen des Themenbereichs „Sprache“ in den eingeschlossen Primärstudien häufig vertreten sind. Während die Eigenschaften der in den Lehrwerken vorhandenen Texte und die Authentizität der Sprache in den Lehrwerkanalysen sehr häufig untersucht wird, ist auffällig, dass auf die Berücksichtigung von kulturell-sprachliche Unterschieden und den Aspekt Aussprache und Intonation nur gering Bezug genommen wird. Weitere und vor allem spezifischere Studien in diesem Bereich, würden vor allem dazu verhelfen, spezielle Bedürfnisse der konkreten Lernzielgruppe wie z.B. kontrastiv potenzielle Interferenzfehler zu berücksichtigen.

Aus der Synthese lässt sich weiterhin ableiten, dass Primärstudien zu den zwei Themenbereichen „Grammatik“ und „Konzepte des Spracherwerbs“ relativ häufig vertreten sind. Die Studien im Bereich Grammatik konzentrieren sich überwiegend auf die Darstellung, Übungen und das Konzept der Grammatik. Untersuchungen zur 
Förderung der Sprachbewusstheit im Kontext der Grammatik sind nur gering vertreten und wären als ein Desiderat zu beheben. Für die Studien des Themenbereichs „Konzepte des Spracherwerbs“ kann zusammenfassend festgehalten werden, dass die AutorInnen sich überwiegend auf die Sprache und den Bereich der Sprachvergleiche beziehen und sich in diesem Sinne jedoch die Untersuchung von tertiärsprachlichen Prinzipien weniger behandelt wird.

In den Primärstudien des Themenbereichs „Visualität“ liegen überwiegend die Funktion und technische Qualität der in den Lehrwerken verwendeten Bilder im Mittelpunkt der Lehrwerkanalysen und weisen auf einen weiteren Forschungsbedarf im Bereich von Möglichkeiten zu kreativer Bildarbeit hin.

Bezüglich des Themenbereichs „Sprachliche Fertigkeiten“, kann an dieser Stelle festgehalten werden, dass in allen Primärstudien zwar die Entwicklung der Sprechfertigkeit thematisiert wird, jedoch das Lese- und Hörverstehen in den Analysen deutlich weniger behandelt wird und nur selten auf die Entwicklung der Schreibfertigkeit Bezug genommen wird.

Die Ergebnisse dieser Metasynthese zeigen außerdem, dass Lehrwerkanalysen zu linguistischen Gegenständen in ihrer Bedeutung für Deutsch als Fremdsprache, zur Vermittlung von Wortschatz und zu Vermittlungskonzepten nur gering vertreten sind. In diesem Sinne werden vor allem weitere Studien im Bereich der Wortschatzvermittlung in regionalen DaF-Lehrwerken als Desiderat gedeutet.

Abschließend lässt sich festhalten, dass weitere Studien in Bezug auf die Nutzung von Medien im Präsenz- sowie Distanzunterricht, Einzelaspekte wie Sprachmittlung, Förderung von Lernautonomie, das Testen und Prüfen von Sprachkenntnissen, die Vermittlung von Lernstrategien in regionalen DaF-Lehrwerken sicherlich eine Bereicherung für den Forschungsbereich leisten würden.

\section{Literaturverzeichnis}

Breitung, Horst/ Lattaro, Elisabeth (2001): Regionale Lehrwerke und Lehrmethoden. In: Helbig, G./ Götze, L./ Henrici, G. / Krumm, H. J. (Hg.): Deutsch als Fremdsprache. Ein internationales Handbuch. Bd.2. (Handbücher zur Sprach- und Kommunikationswissenschaft 19,1-2). Berlin: De Gruyter, 1041-1053.

Çalık, Muammer/ Sözbilir, Mustafa (2014): İçerik analizinin parametreleri. In: Ĕ̆itim ve Bilim, 39(174). 33-38.

Demiryay, Nihan (2016): Authentische Materialien aus der Perspektive von Fremdsprachendidaktik: Überlegungen zur Anwendbarkeit. In: ZfWT, 8 (1), 239253.

Gerighausen, Josef/ Seel, Peter C. (1982): Regionale Lehrwerke. In: Krumm, H. J. (Hrsg): Lehrwerkforschung- Lehrwerkkritik Deutsch als Fremdsprache. München: Goethe- Institut, 23-35.

Gerighausen, Josef/ Seel, Peter C. (1983): Interkulturelle Kommunikation und Fremdverstehen. Dokumentation eines Werkstattgesprächs des Goethe-Instituts. München.

Jones, Michael L. (2007): Overview of Methods. Reviewing Research Evidence for Nursing Practise: Systematic Reviews. London: Oxford Blackwell Publishing. 
Krippendorf, Klaus (2011): Agreement and Information in the Relability of Coding. In: University Pennysylvania Scholarly Commons Department Papers Communication Methods and Measures, 5(2), 93-112.

Krumm, Hans Jürgen (1987): Brauchen wir eine fremdkulturelle Perspektive in der Methodik des Deutsch als Fremdsprache-Unterrichts? In: Wierlacher, A. (Hrsg.): Perspektiven und Verfahren interkultureller Germanistik. München, 267-281.

Miles, Matthew B./ Hubermann, Michael A. (1994): Quality data analysis: a sourcebook. Beverly Hills: Sage Publications.

Möhler, Ralph (2016): Qualitative Evidenzsynthesen- Methodologien, Methoden und Herausforderungen. In: QuPuG. Journal für qualitative Forschung in Pflege und Gesundheitswissenschaft, 2(3), 70-77.

Noblit, George W. / Hare, Dwight R. (1988): Meta-Ethnography: Synthesizing Qualitative Studies. In: Qualitative Research Methods, Vol. II. Newbury Park, CA: Sage Publications.

Osterloh, Karl-Heinz (1978): Eigene Erfahrung- fremde Erfahrung. In: Unterrichtswissenschaft, 8, 189199.

Rösler, Dietmar/ Schart, Michael (2016): Die Perspektivenvielfalt der Lehrwerkanalyse - und ihr weißer Fleck. Einführung in zwei Themenhefte. Info DaF, 5, 483-493.

Sandelowski, Margarete/ Barroso, Julie (2003): Classifying the Findings in Qualitative Studies. In: Qualitative Health Research, 13(7), 905-923.

Tichy, Ellen (2012): Regionale Lehrwerkforschung- Deutsch als Fremdsprache in Ungarn von der Wende bis 2010. Schriftenreihe Lingua Fremdsprachenunterricht in Theorie und Praxis Band 21. Hamburg: Verlag Dr. Kovač. 